Invited commentary

\title{
Enhancing the impact of Artificial Intelligence in Medicine: A joint AIFM-INFN Italian initiative for a dedicated cloud-based computing infrastructure
}

\section{A R T I C L E I N F O}

\section{Keywords}

Artificial intelligence

Decision support systems

Computing infrastructure

Distributed learning

\begin{abstract}
A B S T R A C T
Artificial Intelligence (AI) techniques have been implemented in the field of Medical Imaging for more than forty years. Medical Physicists, Clinicians and Computer Scientists have been collaborating since the beginning to realize software solutions to enhance the informative content of medical images, including AI-based support systems for image interpretation. Despite the recent massive progress in this field due to the current emphasis on Radiomics, Machine Learning and Deep Learning, there are still some barriers to overcome before these tools are fully integrated into the clinical workflows to finally enable a precision medicine approach to patients' care. Nowadays, as Medical Imaging has entered the Big Data era, innovative solutions to efficiently deal with huge amounts of data and to exploit large and distributed computing resources are urgently needed. In the framework of a collaboration agreement between the Italian Association of Medical Physicists (AIFM) and the National Institute for Nuclear Physics (INFN), we propose a model of an intensive computing infrastructure, especially suited for training AI models, equipped with secure storage systems, compliant with data protection regulation, which will accelerate the development and extensive validation of AI-based solutions in the Medical Imaging field of research. This solution can be developed and made operational by Physicists and Computer Scientists working on complementary fields of research in Physics, such as High Energy Physics and Medical Physics, who have all the necessary skills to tailor the AI-technology to the needs of the Medical Imaging community and to shorten the pathway towards the clinical applicability of AI-based decision support systems.
\end{abstract}

\section{Introduction}

Beyond the discovery of the basic principles on Medical Imaging, Physicists have been contributing to the development of instrumentation and software techniques useful for the medical field during the last century [1]. Even today, new technological challenges arise continuously, as a consequence of scientific progress, unmet daily needs or sanitary emergencies.

Since the mid-eighties, Physicists have been collaborating with Clinicians to complement imaging systems with software tools for automating reading of medical images [1]. The initially proposed approaches, mainly rule-based decision systems [2], were mostly replaced by machine learning (ML) techniques in the $1990 \mathrm{~s}$. At that time, the most widely used paradigm for medical image analysis was a pipeline of algorithms consisting of image filtering, identification of regions of interest, hand-crafted feature extraction, and feature classification with ML. Among the ML techniques more-widely implemented, Artificial Neural Networks (ANN) dominated the 1990 s, kernel methods such as Support Vector Machines (SVM) were very popular in the 2000 s, whereas a renewed interest in Neural Networks, especially in Deep Learning (DL) models, characterized the last decade.

Even though the driving application of Artificial Intelligence (AI) in medicine has been represented by the Decision Support Systems (DSS), developed to assist Clinicians in the field of diagnostic imaging especially in large-scale screening programs, a number of other implementations in several different fields of Medicine have arisen in the last 40 years [3-8].

In this work, we will focus on the most recent applications of AI in the Medical Imaging field, including both radiological imaging and radiation therapy [9-12].

Regardless the specificity each development deserves, the currently most widely implemented tasks in the field of automated image analysis based on ML and DL can be grouped into three main categories:

1) image segmentation, i.e. image-to-image conversion, consisting into a pixel/voxel-wise assignment of a medical image into different

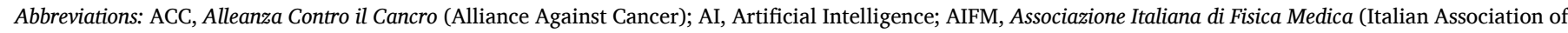

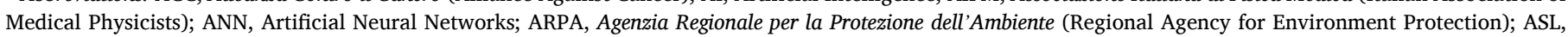

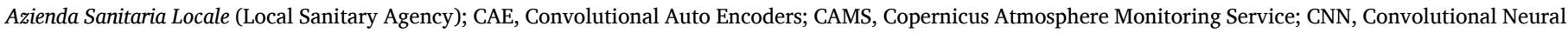

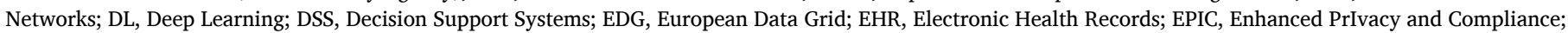

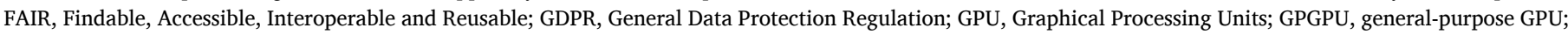

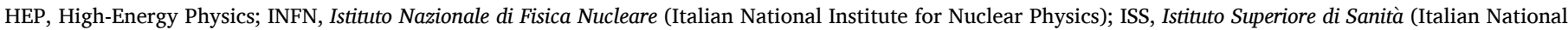

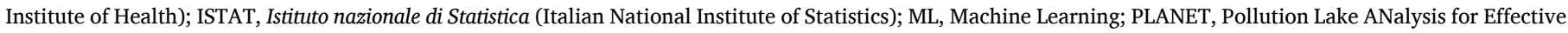
Therapy; QA, Quality Assurance; RT, Radiation Therapy; SVM, Support Vector Machines; XAI, explainable AI. 
regions of interest (such as organs, lesions and background), which is currently efficiently performed by Convolutional Auto Encoders (CAE) and U-nets $[13,14]$;

2) image classification via Radiomics, i.e. image-to-label conversion in two steps, which comprises the image segmentation into regions of interest, then their characterization in terms of Radiomic features [15], generally followed by a ML- or DL-based predictive models [16];

3) image classification via DL, i.e. image-to-label conversion in a single step, which is completely data driven, and implements DL methods, such as Convolutional Neural Networks (CNN), to directly analyse medical images and assign them to a diagnostic/prognostic category without the need for specific preprocessing $[6,17]$.

Each task can be accomplished with different methods, having different strengths and weaknesses, and the choice among the different approaches depends on the segmentation/classification problem to be solved, and also on the need to have a fully interpretable analysis pipeline [18]. Nonetheless, all the methods mentioned above are typically characterized by an extremely large number of free parameters, and thus they all need large amounts of annotated data to be properly trained.

Despite recent advances in the development of $\mathrm{ML}$, DL, and the ability of computers to handle large amounts of data, the integration of these technologies into clinical workflows and their application to personalized medicine requires additional research and development efforts. Pilot studies conducted in single centres, and in many cases with limited amounts of data, are certainly necessary to highlight new problems that can be faced with AI-based solutions. Nonetheless, largescale validation to probe the generalization ability and the reliability of AI-based systems, together with further developments to favour the interpretability and explainability of their outputs are strongly needed to make these solutions acceptable by the medical community and applicable in the clinical routine.

An agreement between the Italian Association of Medical Physicists (Associazione Italiana di Fisica Medica, AIFM) and the Italian National Institute for Nuclear Physics (Istituto Nazionale di Fisica Nucleare, INFN) was signed to strengthen the synergy between the two fields, which constitutes a fruitful groundwork for interdisciplinary interchange and development with stimulating prospects, including the possibility of initiating a rapid diffusion of effective and reliable AIbased clinical tools. One of the actions proposed in the field of AI is reported in this paper.

This paper aims to investigate the possibility of overcoming the current barriers that prevent the development, large-scale validation and reliability assessment of AI-based DSS relying on complex multimodal heterogeneous patients' data. Easy-to-use technologies to efficiently handle and mine the Medical Imaging Big Data need to be developed, consolidated and made available to the Clinics to shorten the pathway towards precision Medicine. We suggest the synergy between the Medical and High-Energy Physics research fields as a fruitful strategy to achieve efficient practical solutions to overcome the current technical obstacles and to lead to a significant step towards the reliable, qualityassured and efficient implementation of AI-based solutions in the medical field.

This paper is structured as follows: the expected advantages of the use of AI-based tools in several fields of Medical Imaging are highlighted in section 2; then, the main current barriers that prevent the use of AIbased solutions in clinical workflows are discussed in section 3; finally, a strategy synergically developed by the Medical and HighEnergy Physics communities to speed up the translation of AI-based research tools into the Clinics is presented in section 4.

\section{AI potential impact on medical imaging workflows}

Medical Physicists within the Hospital are fundamental not only to maintain a uniform standard of services for all patients but also to face the new challenges ahead. The rapid development of new information technologies represents a strong incentive to use decision-making systems based on AI in Healthcare and many studies are already available describing applications to diagnose and treat diseases or epidemics and manage their socio-economic impacts.

AI, when used appropriately, can be a powerful tool to aid Physicians in making diagnosis by correlating clinical and imaging data, in monitoring patients using imaging, in optimizing the management of Healthcare spaces (Intensive Care Units) or in the coordination of regional networks for the on-site management of patients.

Machine learning and deep learning algorithms can change the clinical workflow in almost all the fields of Medicine from Diagnostics to Therapy and quality assurance. Interdisciplinary work between different professionals is necessary to reach the goal, and Medical Physicists can play an important role since they are a bridge between technology and medicine. Many reviews are available in literature describing the AI applications in Medical Imaging, Radiation Therapy (RT), Quality Assurance (QA) fields, also describing the role of Medical Physicists [1,19-22].

One of the first applications of $\mathrm{AI}$ in the medical field is radiological imaging due to the fact that patients' images are complex, and it is difficult to discriminate, detect and classify lesions on them [23]. In fact, both normal tissue and tumour volume can vary and show different characteristics, therefore it is fundamental to use a robust analysis method [24]. In this field, ML applications have increased with the coming of DL and a boost was impressed in segmentation, detection and classification of the lesions, but also in imaging reconstruction, artifact reduction and to estimate the dose delivered to the patient $[23,25]$.

AI has also strongly entered Radiotherapy in all steps of the patient's workflow to ensure the best treatment for the patients [26]. The boundaries between diagnostics and treatment have been mitigated, thus, many AI applications are also used in Radiotherapy. A large amount of images are produced in Radiotherapy from the diagnostic images to the treatment ones as well as those acquired during the Adaptive Radiotherapy to automatically check lesion position during the radiation delivery through patient positioning images [27]. ML and DL applications are used in treatment planning systems, to evaluate the treatment response, to follow the tumour during the treatment, and to evaluate the dose delivered to the patient. AI systems are also available for clinical DSS for dose adaptation [25].

In addition to the optimisation, also the QA and the patient-specific dosimetry can be improved by utilising AI methods [28]. AI approach to QA led to the development of more performing systems helping Medical Physicists to control more advanced equipment [29].

As deeply discussed in a number of recent reviews [30,31], the fields of application for AI-based DSS in Healthcare are wide and diverse, with a high potential to lead to more accurate patients' diagnosis, shorten the diagnostic pathway, efficiently monitoring the treatment courses and possibly predicting a treatment outcome, in most cases leading also to a reduction of the costs of the Healthcare service.

\section{Current limitations to the use of AI-based solutions in clinical workflows}

Despite Medical Imaging has long been approaching the Big Data Era for a while, efficient strategies and infrastructures to organize and mine the every-day-growing resources of knowledge are still lacking. This is one of the challenges to face [19]. Collecting Big Data samples is not a straightforward task, both because they should be large enough to be representative of the population affected by the specific pathology to be investigated, and mainly because for training and validating an AI-based DSS, data should be carefully annotated and continuously updated. This operation requires the use of valuable time by medical experts. Due to the huge effort required to collect and annotate meaningful datasets, it would be utmost important to make previously collected data Findable, 
Accessible, Interoperable and Reusable (FAIR) [32].

In addition to efficient strategies for data collection, reuse and sharing, another fundamental ingredient to develop a DSS is the availability of a well-equipped computing infrastructure to train the AI-based models, and to make extensive validations of the performance achieved on new, previously unseen data. Training a DL algorithm requires specialized hardware, such as general-purpose GPUs (GPGPUs), adequately large RAM, and fast access to storage. A computing platform devoted to the secure collection and analysis of clinical and imaging data of subjects was developed by Retico et al. to support the interdisciplinary research aiming at the identification of a neuroimaging-based biomarker of Autism Spectrum Disorders [33].

Similar computing infrastructures are not readily available neither in Hospitals, nor in most research departments.

Generally speaking, once a DSS system is successfully developed and validated in a research contest, before it can be applied in a clinical setting a number of verification steps should be carried out. Among the fundamental requirements a DSS has to satisfy, a reliability check is mandatory [34]. Even in case a software application obtained the approval by the regulation committee for its use as DSS, a protocol for a continuous verification of the stability of its performance should be set up. DSS performance indeed generally depends on the quality of input data. Dedicated protocols should be set up for quality assessment of a DSS [35].

From the practical point of view, the main current limitations to the use of AI-based DSS in clinical workflows can be summarized as follows:

1. absence of suitable AI-based DSS solutions for a specific clinical problem of interest;

2. absence of accurate and large-scale evaluation of the specific DSS of clinical interest;

3. absence of extensive data samples (either mono- o multi-centre data) either to develop or to evaluate the performance, the reliability and the reproducibility characteristics of the DSS of interest;

4. absence of adequate computational resources and technical expertise to eventually train or customize via transfer learning a new DSS for a specific clinical problem of interest;

5. absence of a validation protocol to make a QA evaluation of the DSS performance before it is used in clinical studies and to guarantee the stability of its performance;

6. absence of a clear interpretation/explanation for the DSS model response.

In a typical scenario, a Clinician believes that the use of a DSS would be beneficial in accelerating the clinical workflow for a specific diagnostic/treatment task, but no suitable tools do exist (point A). Hence, he/she would instruct the Medical Physicists to provide a technical solution. The Medical Physicist might find, either on the market or made publicly available by the scientific community, a suitable DSS for that purpose, and he/she would find it appropriate to validate the system on a large-scale data sample, which may be neither available nor easy to collect and annotate (point B). Conversely, in case the Medical Physicist finds that a suitable DSS does not exist, a joint research project involving Clinicians, Physicists and Computer Scientists may be carried out to develop such DSS. Then, the absence of a suitable data sample to develop the system could be a strong limiting factor (point $\mathrm{C}$ ). A pilot/ discovery study can be set up for the development of a custom AI-based solution for that specific task in case a sufficiently large and annotated dataset is available, even if it was acquired in a single medical centre. It may happen that, even in case suitable data samples are available to develop a custom DSS, its development is hampered by the absence of either adequate computing resources, technical knowledge on data mining and AI, or remote computing resource handling (point D). In the lucky case where all hindering issues A-D of the list above are overcome, issue E shows up anyway. It is up to a Medical Physicist to implement a QA programme of each DSS to be used in clinical workflows, thus, specific QA protocols for AI-based DSS should be set up. Finally, most DSS systems developed so far, especially if they are based on complex ML or DL models, are barely interpretable to humans and the motivations behind their response cannot be easily explained to patients (point F). DSS systems often act as "black boxes", thus preventing the necessary confidence in their use from being built. Further research in the field of explainable AI (XAI) is mandatory to match the requirement to have DSS systems with high performance, which are generally complex and barely interpretable, with the need of providing responses that can be explained to patients $[18,36,37,38,39]$.

\section{A synergic solution by Medical and High Energy Physics community: The approach proposed by AIFM and INFN}

The development and validation of AI-based techniques for Medical Physics applications, ranging from diagnosis, optimization of resources, and therapeutics, share the need for non-trivial computing infrastructures, such as:

- computing intensive facilities, with specialized hardware (i.e. GPGPUs), where to address the expensive AI algorithm training;

- storage systems able to store large datasets from epidemiological studies and from complex measurement devices;

- the capability to be pervasive on the territory, joining data from multiple sources and centres;

- a strong focus on privacy related issues.

The effort needed to put in place such an infrastructure is well beyond the possibilities of single medium and even large centres; the added complexity of privacy themes (as those granted by the General Data Protection Regulation, GDPR) adds a further layer of complexity [40].

Other fields of science and technology have already been facing similar issues (with maybe less focus on privacy); it is then worth exploring the solutions deployed and their applicability to Medical Physics studies.

The INFN is a pioneer in the design, deployment and operations of large-scale computing infrastructures, mainly developed in order to fulfil the need of the last generations of High Energy Physics (HEP) Experiments. INFN has been a core and founding member of projects like the European Data Grid (EDG) [41], EGEE [42], INDIGO-DataCloud [43], EOSC-hub (https://www.eosc-hub.eu/), ESCAPE (https://proje ctescape.eu/) and many others, which have revolutionized the scientific computing since the first years of $2000 \mathrm{~s}$.

INFN has deployed a complex computing infrastructure, with resources in excess of two hundred Petabytes (PB) of storage, and computing facilities with a total of more than 100,000 computing cores. The resources are distributed in 10 facilities geographically distributed on the Italian territory, with the centre in Bologna (INFN-CNAF) deploying nearly half of the total (https://www.cnaf.infn.it/en/).

The HEP experiments (and a new generation of data intensive experiments, in Astroparticle and Applied Physics) are operating a decadelong transition to Cloud-inspired distributed architectures, in which resources can be centrally steered and organized independently of their location. The INFN-Cloud project, launched in early 2020 and currently in production, is the driving force for the Cloud development for all the INFN initiatives.

Such an infrastructure, when matched with the pervasive high-speed networking offered by the Italian national research and education network (GARR, https://www.garr.it/en/) already connecting most of the clinical research sites and all the INFN facilities, offers a technical solution for data and computing intensive research in Medical Physics in Italy. 


\section{The INFN-Cloud initiative and capabilities}

INFN-Cloud is a multi-site, multi-organization federated Cloud infrastructure that provides a portfolio of Cloud compute and storage services available via user-friendly portals, but also exploitable via command-line interfaces and APIs. From the architectural perspective, INFN-Cloud has been built on two main pillars: distributed resource orchestration and a modern federated solution for industry-grade identity access management capable of managing both authentication and authorization. These are the key elements to federate multiple Cloud infrastructures and computing centres.

The portfolio of the INFN Cloud services is based on open-source software and on de-jure or de-facto standards, following the services composition principle. This is implemented through a declarative mechanism, allowing users to care about the "what" instead of the "how" to autonomously create their own solutions. All this makes the portfolio highly modular, dynamic and ready to be adapted to new user requests.

Although the main objective of INFN-Cloud is to support Scientific Computing, it offers storage and compute capabilities to create and run scalable applications in a dynamic environment, coping with the needs of a huge variety of use cases, including those that require the processing of sensitive data (for Medical Physics or other fields) in a trustable way.

Since it offers support to both traditional silos or Data Warehouse approaches, as well as to more advanced methodologies such as the Data Lake [44-46], INFN-Cloud is perfectly suitable to deploy platforms to collect and process heterogeneous datasets that could also include unstructured data. In this respect, INFN-Cloud supports also storage-based event platforms for automating metadata processing workflows as well as data pipelines.

The federation of data, resources and solutions is a peculiarity that represents a key added value of INFN-Cloud, allowing third-party providers to be included in the very same computational environment, while preserving, if necessary or required, the ownership of the underlying resources and data. This allows to effectively support data coming from multiple sources, according to the many use cases presented by data producers and consumers.

As an example, a third-party contributor could be a Clinic or Hospital that needs/wants to perform some type of local data processing involving, for example, the anonymization of the data, and that wants to federate its resources with the data lake, distributing this "pre-processed data" for further analysis and exploitation by other users or communities.

\section{Privacy oriented capabilities in the INFN Infrastructure}

Treatment of Electronic Health Records (EHR), medical images and personal data in general (for example user account data) requires an additional level of organization in order to protect data subjects' fundamental rights and freedoms. In particular, Data Controllers such as Clinics and Hospitals, need to be able to demonstrate their compliance to GDPR and other laws and regulations concerning personal data and cybersecurity. In case Data Controllers decide to leverage an external cloud provider for the analysis of personal data, they "shall use only processors providing sufficient guarantees to implement appropriate technical and organisational measures in such a manner that processing will meet the requirements of this Regulation and ensure the protection of the rights of the data subject" (GDPR art. 28). INFN-Cloud provides such guarantees through its certification ISO/IEC 2700127017 27018, a set of word wide accepted information security compliance frameworks. These frameworks are adopted in the Enhanced PrIvacy and Compliance (EPIC) Cloud partition of the INFN-Cloud federation.

In practice, the certification implies to yearly demonstrate to independent third-party auditors to have put in place a set of functions, processes, controls, systems, procedures and technical measures in order to protect, make available and secure the information processed and in order to implement data protection and security by design and by default. A detailed description of the EPIC Cloud information security measures is out of the scope of this paper. We briefly list, for example purposes only, some of the most relevant ones:

- identity and access control management system capable of granting and modifying access rights based on the "least-privilege" principle and on the "need-to-know" principle;

- segregation of duties enforced at all architectural levels;

- risk based approach to information security, that is high risk data and processing activities are assessed. Then preventative measures to mitigate risks are defined and implemented;

- business continuity plan defined and regularly tested;

- robust policies and procedures defined and followed by all personnel;

- due diligence checks on suppliers performed regularly in order to verify their safety and protection capabilities;

- encryption at-rest and in-transit (AES-256-GCM, ChaCha20Poly1305, HMAC-SHA-256 pseudo random function, TLS v1.2+);

- secure key management system and procedures;

- secure disposal of obsolete devices;

- cyber security policies covering regular vulnerability and malware scans, patching, updating, account checking, Access Control Lists and firewall checking;

- organizational processes and security information and events monitoring system (SIEM) automating the management of security events and enabling for timely data breach notification (Art. 33 GDPR);

- clear definition of organizational roles and responsibilities;

- detailed logging and auditing information gathered in order to ensure accountability.

The subjects participating in a clinical trial will need to obtain permission from the Ethics Committee for the clinical research involving patient data and their export to an external infrastructure for analysis.

In order to comply with GDPR, the users will be required to pseudoanonymize the data before being processed. The data will be required to be collected with written consent, where the scope of data storage and processing is described, and the authorization from the local Ethics Committee will be required to be readily available upon request. Personal data will be deleted from the repository after the end of the clinical trial or analysis.

The EPIC Cloud is already being successfully exploited by various use cases dealing with genomic or clinical data:

- Alliance Against Cancer (Alleanza Contro il Cancro, ACC) (https://www.alleanzacontroilcancro.it/en) who pursue clinical and translational research in order to bring state of the art diagnostics and advanced therapeutics to patient care. At present the ACC platform hosts radiomic images of 2500 patients (DICOM, DICOM-RT files) and genomic data of 1000 patients (BAM and VCF files).

- PLANET (Pollution Lake ANalysis for Effective Therapy, INFNfunded research initiative), an observational study aimed at assessing a possible statistical association between Covid-19 infection, symptoms, course on one side and environmental pollution on the other side.

- Harmony Alliance (https://www.harmony-alliance.eu/), the European Public-Private Partnership for Big Data in Hematology, deployed his data platform on EPIC Cloud. Today about 60,000 patients' genomic datasets (BAM, FASTA, FASTQ and VCF files), coming from 140 organizations from all over Europe are being stored and analysed.

\section{A cloud design for large scale medical research on INFN Infrastructure}

INFN and AIFM are proposing a Cloud-based infrastructure designed 


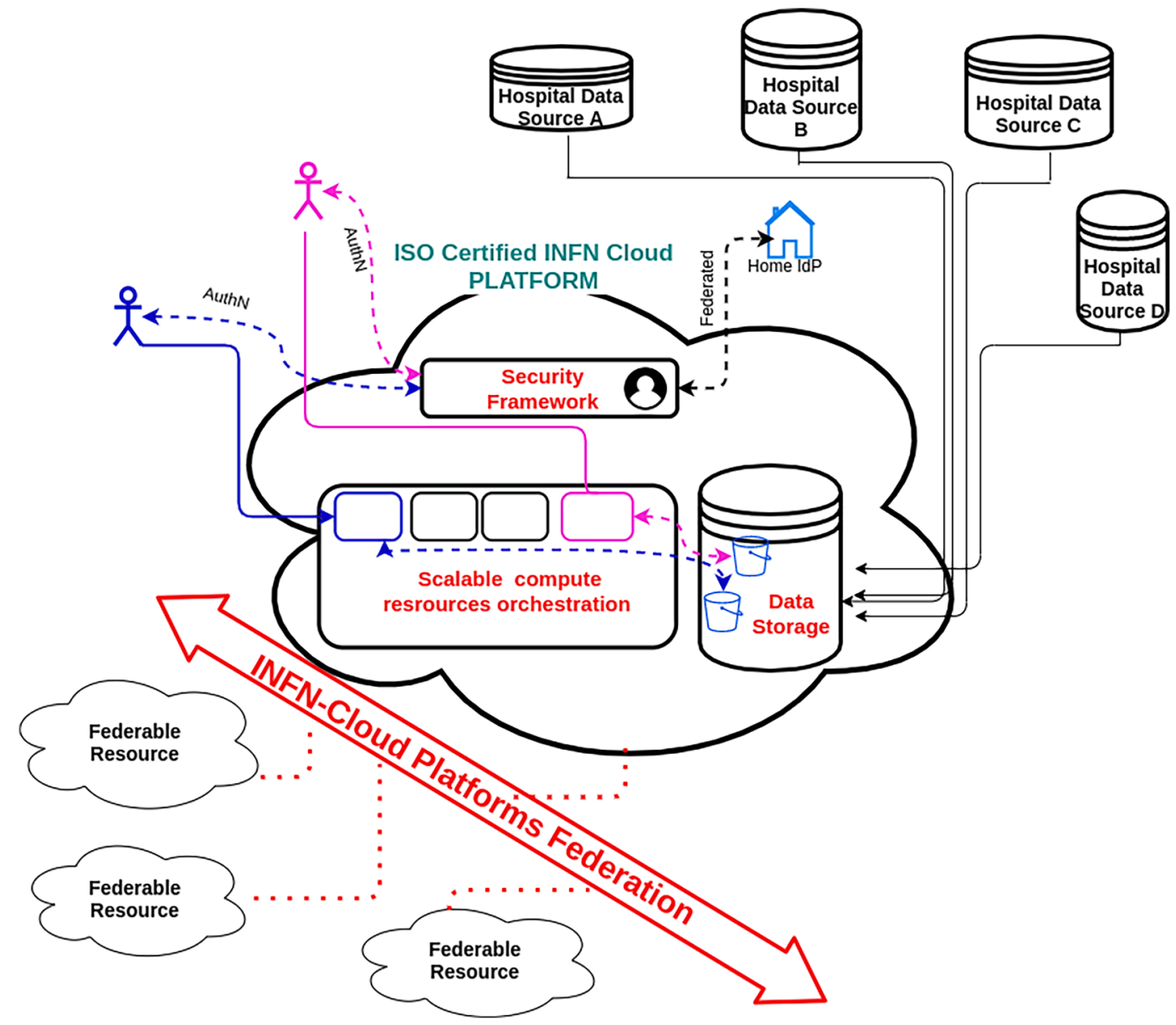

Fig. 1. The INFN-Cloud design, explicitly showing the capabilities to access external data sources and federated resources.

Table 1

A partial list of the tools available via INFN-Cloud and in the portfolio of INFN solutions.

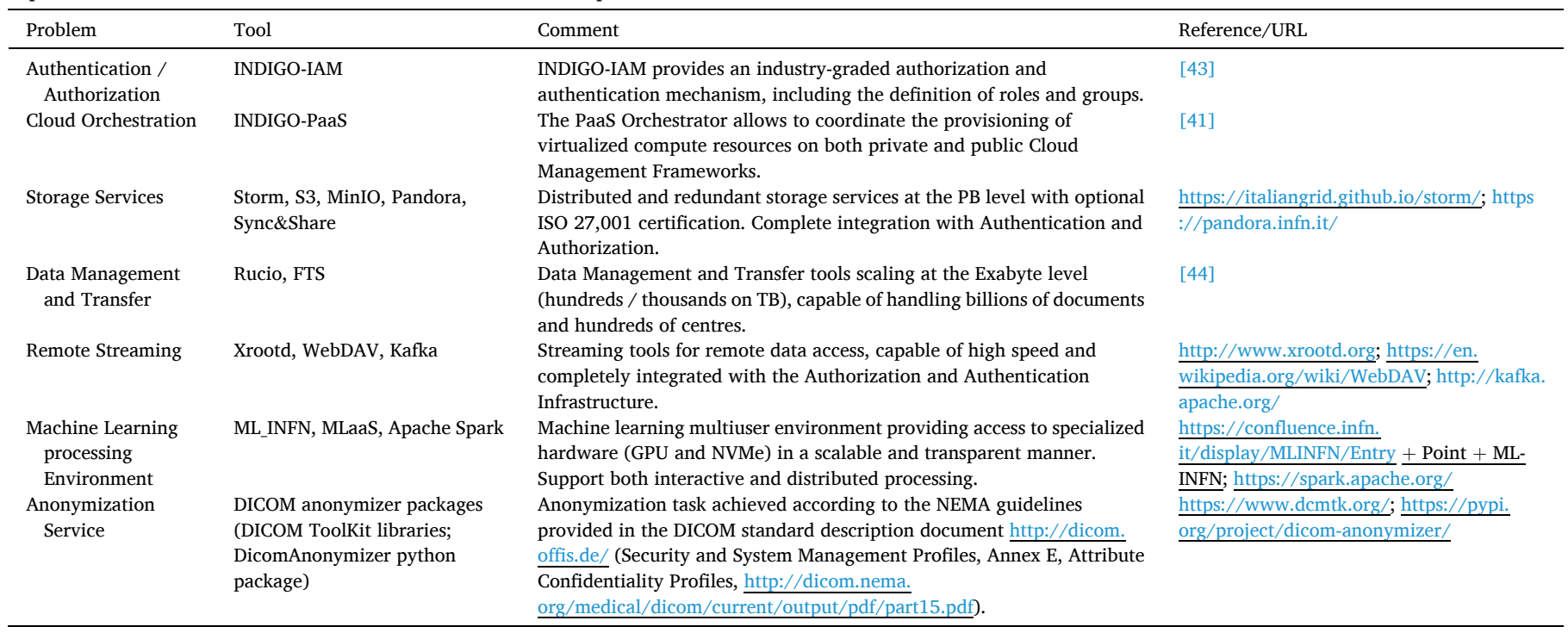

for AI oriented data and computing research in Medical Physics, with:

- a geographical disperse organization on the territory, with the capability to be pervasive at the level of single Hospitals and Clinical Research Centres;
- the capability to present a single logical view of the infrastructure, and hence be steered centrally when aggregation multi source data sources;

- a ground-up privacy-oriented design, able to satisfy the diverse needs of clinical centres and initiatives; 
- data management according to the FAIRness principles [32].

The partitioning of resources (data and computing) between local and global environments allows to limit the visibility or scope of the data, a desired feature when dealing with sensitive data; at the same time, the global logical view allows to steer processing and data movement centrally, with a reduced need for expert staffs at clinical centres.

The platform design, sketched in Fig. 1, allows for implementation of different solutions, which are based on the technical modules listed in Table 1; they are detailed in the following.

\section{Solution 1: harvesting of data collected at clinical facilities}

In this type of workflow, data collected and/or generated at clinical centres are harvested and moved to the INFN infrastructure, maintaining the high level of privacy as provided by the INDIGO-IAM Authorization and Authentication framework and by the ISO/IEC 27001 storage certification. Access, further transfers and deletion can be operated only by specific roles; in this way, the data owner can use the processing power from INFN facilities while still being completely in charge of the data lifecycle or decide to anonymize the data via suitable tools that process DICOM files and make it available to larger subsets of users (eventually all, in public form).

The harvesting can be operated in different ways: INFN-Cloud operated nodes can be installed at the facilities, with remote administration and thus a low impact on the operations of the local personnel or can be injected in INFN-Cloud storage from the hospital/centre storage system via edge services with minimal maintenance (for example, using Web services via simple browser).

\section{Solution 2: Local Anonymization}

Whenever the harvesting of sensitive data is not deemed feasible by the ethics committee of a given research not even if shielded by the INDIGO-IAM security infrastructure, we plan to be able to deploy, locally at the clinical facility, anonymization and hashing services (either in the form of standalone services or steered by the Cloud central services). With these, data can be anonymized by using industry standard techniques, with the hashing database remaining locally to the centre. In such a way, data injected to the INFN storage can have the additional level of security on top of ISO/IEC 27001.

\section{Solution 3: Local Feature Extraction}

In many AI inspired workflows, such as those based on Radiomics approaches $[15,16]$, the initial raw data from instrumentation needs to undergo a preprocessing phase, in order to extract features and ease the subsequent training process. The output feature, loosely related with the initial data, may or may not be needing further anonymization. The solution INFN and AIFM are planning involves the capability of preprocessing on hardware local to the data collection centre, either via locally deployed services, or steered via the INFN Cloud central services. Output features can then either be harvested directly to INFN Cloud storage endpoints, or be anonymized, or be further processed locally.

\section{Solution 4: Distributed Learning}

An interesting development in Machine Learning when interfaced with large training datasets is the capability to distribute processing to remote sites, holding a fraction of the total input data (see for example https://keras.io/guides/distributed_training/). This would turn out to be very beneficial in medical research, since it would allow the sensitive input data not to leave the collection centre, while still allowing for a training procedure on the whole dataset. Successful examples in this direction have been provided by Jochems et al. (2016) [47] and by Deist et al. (2017) [48]. From the hardware point of view, the availability of powerful Machine Learning oriented workstations (GPU equipped) at low price allows for efficient operations even without large and difficult to operate computing centres. From the privacy point of view, the original sensitive data never leaves the initial centre, and the output trained network is by no means usable to derive any characteristics on them.

The preparation, maintenance and operations of such a local service is not trivial and it needs specialized manpower; the Cloud solution INFN and AIFM are suggesting overcomes this problem as:

- the local hardware is installed as a leaf of a Distributed Cloud setup, and hence the applications are installed, manager and operated centrally;

- no application is allowed to move data over the network; it can only be accessed by the Machine Learning application, which uploads only configurations.

After the training phase, the algorithm is made available both locally (evaluating local data, again not distributed over the network) and globally, for example operating on a public database.

\section{Usage examples for the proposed cloud-based infrastructure}

Potentially exciting new applications that could benefit from AIbased solutions come to light every day. Clinicians, Medical Physicists and Computer Scientists are continually looking for solutions to specific problems that may require the customization and refinement of existing analysis techniques or the development of new ones. The Cloud-based infrastructure proposed in this paper is expected to facilitate and accelerate this process.

As a selected pool of usage examples, we propose a few applications for each of the four technical solutions described above.

\section{Case studies for Solution 1}

A specific operation in image analysis that requires all original images to reside in the same physical place is the iterative building of a population-based template, which requires the coregistration of each image to a temporary template and the generation of a new template by averaging all coregistered examples [49]. From the ML point of view, during the development of a novel classification algorithm based on multicentre data, harvesting data in an individual repository can be maximally efficient for model training, validation and testing. In this case, data collected at different sites can be handled during the model fitting in order to teach the model to get rid of the possible confounding information related to the data acquisition site [50].

\section{Case studies for Solution 2}

According to the Ethical Committee approval specifications for each research study, the possibility of harvesting data can be limited to fully anonymized information. Solution 2 will allow the execution of all necessary anonymization procedures (both on DICOM metadata and directly on images, if necessary) at the acquisition site.

\section{Case studies for Solution 3}

In many multicentre studies based on Radiomics and ML, it can be unnecessary (and sometimes not allowed by the Ethical Committee) harvesting huge data cohorts in a central repository. It can be very useful to be able to execute locally at each site the data processing to extract the samples of radiomic features, then ML algorithms can be trained on the pooled data cohort. This is the approach adopted by a world-wide research collaboration in the field of neuroscience, the ENIGMA Consortium, which collects brain features extracted from MRI images at each contributing site according to a predefined analysis protocol which includes also a data quality check [51].

\section{Case studies for Solution 4}

In case neither the original image data nor the derived features can be moved outside of the Hospital, according to specific disposals by the Ethical Committee, the proposed solution of distributed learning can allow the use of these data for ML model training and validation. This 
modality is certainly extremely useful even in case a DSS has to be customized on characteristics of data acquired at a specific site. A transfer learning protocol can be set up in this case to retrain the final layers of a DL-based DSS trained on different data sources on site specific examples to optimize the DSS performance.

\section{Practical implementation example for the proposed INFN-AIFM infrastructure}

Although the proposed AIFM-INFN cloud-based infrastructure is still in the design phase, all the components necessary to implement it have already been technically developed within the INFN community and successfully implemented also in practical analyses of medical data. Two specific and complementary examples of AI-based analysis approaches will be described below, both conceived and implemented in response to the COVID-19 pandemic.

1. Development of an AI-based analysis pipeline for the automated quantification of the percentage of lung parenchyma affected by lesions related to COVID-19 pneumonia on chest CT scans, developed within the INFN-funded AIM project [52]. To carry out the automated analysis of the CT scans, a processing pipeline constituted by a sequence of two U-nets [51] has been set up taking advantage exclusively of publicly available annotated data samples of lung CT acquired in different clinical centers. Nearly 1800 CT scans were collected in a central database, each scan being a few tens of MB depending on the size of patients. To train the first U-net, devoted to lung segmentation, nearly $500 \mathrm{CT}$ scans were used. For the second Unet, devoted to COVID-19 lesion segmentation, about 250 CT scans were available. A U-net is a fully-convolutional neural network which operates an image-to-mask conversion. To identify complex structures, such as the lungs and the COVID-19 lesions, the network architecture required six levels of depth, thus saturating the $16 \mathrm{~GB}$ RAM limit of the available GPUs. Fast access to storage was also necessary during the training phase to dynamically access the examples which could not simultaneously be kept in memory.

2. AI-based study of possible statistical association between air pollution and Covid-19 developed within the INFN-funded PLANET project. The study is based on the hypothesis that pollution may contribute to the spread and/or the severity of COVID-19, through 2 possible mechanisms:

a. Acute: microparticles derived from fossil fuels might act as airborne carriers of virus;

b. Chronic: exposure to microparticles and chemical pollutants might cause chronic lung injury, exacerbating the consequences of viral infection.

A key element of PLANET is to include a wide variety of components that are expected to influence rates of SARS-COV-2 diffusion and infection such as atmospheric data, population density, urban vs rural environment, mobility, socio-economic conditions, etc. and at the same time the project aims to take into account also the fact that severity COVID-19 disease and deaths are also influenced by many variables (age, gender, comorbidities, frailty, etc..).

To this end data from several sources have been collected, namely: data from the Regional Agency for Environment Protection (Agenzia Regionale per la Protezione dell'Ambiente, ARPA) of Umbria region (both sensors and models); both data from Copernicus Atmosphere Monitoring Service (CAMS) and Climate data; the Italian National Institute of Statistics (Istituto Nazionale di Statistica, ISTAT); Deprivation Indexes; Epidemiological data. The latter currently includes: Data from the Italian National Institute of Health (Istituto Superiore di Sanità, ISS); Data from the Local Sanitary Agency (Azienda Sanitaria Locale, ASL) of Viterbo (IT); Data from Liguria region. Personal data received by ISS and ASL have been managed in the context of the organizational framework of the EPIC cloud, which ensures that the right agreements are in place in order to comply with GDPR as both Data Controller (ISS data) and Data Processor (ASL Viterbo data). All the above-mentioned sources populate the PLANET DataLake where not only the data are made accessible to the analysts, but it also offers a tightly integrated computer platform enabling an effective analysis flow. An ongoing activity is moving the PLANET platform inside the EPIC environment. In practice this means to harden the current platform applying the technical measures required to improve the security posture of the whole system.

The first example above shows a typical application highly demanding in terms of computing resources, which is barely feasible if not impossible without dedicated and efficient computing infrastructure equipped with GPUs. The second example describes the capability to aggregate data (not including heavy imaging data at present) from multiple sources in a privacy-oriented design (EPIC infrastructure where the PLANET Platform will be hosted), which is particularly suitable for handling medical data.

Putting together the efficient computation capability demonstrated in example 1 and the appropriate sensitive data handling and capability to view different clinical centers as a unique logical entry demonstrated in example 2, it is apparent that all technical ingredients necessary to build the INFN-AIFM cloud-based computing infrastructure mainly dedicated to medical image analysis with AI-based techniques are almost ready to use. From the technical point of view, further developments will be needed to interoperate the computing resources within the data platform of EPIC cloud, and, relying on the collaboration with AIFM, particular attention would be paid to making the infrastructure compliant both with GDPR requirements and with the principles of data FAIRness.

\section{Discussion}

The collaboration among Medical Physicists, Physicists working in different fields of research involving Big Data and Computer Scientists could provide a valuable contribution across all necessary development and validation steps to be completed before AI-based DSS can be reliably and efficiently integrated in the clinical workflows. Other professionals, mainly with expertise in (Bio)Engineering and Statistics, also clearly have a relevant role in the fields of research involving the acquisition and analysis of biomedical data. Nevertheless, Medical Physicists, Physicists and Computer Scientists have their specific roles in the ongoing transition of medical imaging towards AI, as specified in the dedicated subsections below.

A fundamental aspect of this transition is the Ethical dimensions of using AI in Healthcare [53,54]. AI-based applications are often cited as enabling technologies towards precision medicine $[55,56]$. The discussion of this topic requires a specific expertise that goes beyond the technical skills of the authors of this paper.

Similar initiatives have already been proposed in a variety of medical domains. These projects are in the fields of Radiology, Radiotherapy and encompass all areas of healthcare where there are large amounts of clinical data and images. They work on clouding and data/sharing and/ or distributed learning solutions using on-line platforms, like Amazon, Google which are starting to play a crucial role also in the healthcare field.

One of the most recent applications is AIFORCOVID Imaging Archive. This platform is based on Amazon Web Services (data storage, management and transfer) to develop innovative AI methods for predicting clinical developments of the disease caused by SARS-CoV-2 (https://aiforcovid.radiomica.it/).

Moreover, Stanford University developed a smartwatch-based COVID diagnosis app, in partnership with Amazon, that analyzes elevated heart rates and other abnormalities for pushing real-time alerts to patients suspected of COVID infection. Amazon has offered millions in cloud computing credits for similar diagnostic solutions for digital health innovators across the world (https://www.cio.com/article/ 
3619551/ai-in-healthcare-the-tech-is-here-the-users-are-not.html).

Even Google has announced a series of partnerships with healthcare enterprises: Mayo Clinic will work together with Google Health's AI, in the medical image segmentation. In addition to AI, the two companies plan to use Google Cloud and data analytics to advance the diagnosis and treatment of diseases (https://www.fiercehealthcare.com/tech /mayo-clinic-google-announce-ai-effort-to-boost-radiation-therapy-for -cancer).

There is a collaboration between Varian and Google to build an advanced artificial intelligence (AI) based diagnostic platform (https://www.varian.com/about-varian/newsroom/press-releases/var ian-and-google-cloud-collaborate-aid-fight-against-cancer).

A Google Cloud AI Platform's NAS technology is used to create an AI segmentation engine to create customized auto-segmentation models for organs in the body. These models will be incorporated into Varian treatment planning software tools for use in cancer centers around the world. An ever-increasing number of companies, from start-up to large medical system companies, up to technology giants (Nvidia, Intel, Amazon, Apple, Facebook, Alphabet, Microsoft), are present on the market proposing analytics platforms and/or processing software with the most advanced AI tools. These systems, generally on a cloud-based infrastructures and often freely usable by the individual healthcare professional, are focused to improve disease screening, diagnosis of various diseases, patient therapies and medical operation planning, as well as to share and make available advanced data mining instruments. Not providing for the possibility of local installation in a single hospital, vendors supply their AI services by using cloud systems that can be in one or more countries, sometimes even outside Europe. Typically, these companies have developed AI systems in collaboration with important universities and/or private hospital consortia, the web pages are full of these announcements, either to understand what kind of support clinical practitioners would expect, or to have available the necessary Big Data for training and validating their algorithms. These partnerships were also necessary to address the registration process of these AI medical devices as required by the regulatory bodies (FDA, CE mark) and by the health authorities, as well as well regarded by leading medical-imaging scientific associations (RSNA, ESM, EANM, ESTRO, EFOMP, etc.).

In such a scenario, a platform such as the one created by INFN, equipped with advanced AI tools that can be shared between the different centers on a national level, stands out for its uniqueness, expanding the current functions and moving towards new areas of service not yet available. Moreover due to the intrinsic no-profit INFN character, the platform could result in of facilitated accessibility with lower costs. Since only registered (FDA/CE) medical device AI systems can be used in the clinic, whose effectiveness has been validated on limited data sets, within research projects or pilots that almost never are based on European or Italian experiences, to have a common platform on which to test these AI algorithms is important. The INFN platform, fully responding to the European and Italian regulations in terms of safety and GDPR requests, which although valid for all European countries are applied differently in individual countries, sees the possible storage of healthcare Big Data for their deep analysis and/or the development of optimized and standardized AI algorithms based on the medical knowledge and skills of Italian clinicians and of our national healthcare system. Specifically, it would be used for creating common quality assurance tools, perhaps using techniques of federated learning to reduce patient's imaging sharing, similar on the national territory, to be used for studying safety, robustness, sensibility and specificity, ethics of commercial AI marked tools presents on the Italian centers. Such a platform could also securely integrate with regional health record data, as well as image databases (public and private) on the web, broadening the spectrum of available data, as well as providing a tool for helping ISS to monitoring the correct functioning, effectiveness and the diffusion on the territory of AI-based medical devices.

\section{The role of Physicists and Computer Scientists}

Physicists and Computer Scientists working within INFN-funded research project over the last two decades have developed a number of prototypes of AI-based DSS across several pathological conditions and imaging modalities $[57,58,59,60,61,62,63]$. In most cases, the starting point for developing a new application was an exploratory analysis carried on small data samples, which was later on consolidated and validated on larger data samples.

Physicists working in fields of research involving Big Data, such as the HEP field, where experiments to investigate the fundamental constituents of matter generate an incredible amount of data to be sometimes processed in real time (e.g. the CMS experiment at CERN [64,66], with almost 100 PB of data collected in the period 2010-2018) are currently implementing AI-based solutions in their analysis $[65,67]$. The computing infrastructures, the secure data storing and sharing protocols they are developing for HEP applications can be customized for their use in the medical imaging domain. In addition to technical solutions regarding efficient computing infrastructure HEP Physicists and Technologists can provide, their contribution is expected to be valuable also in building innovative ML and DL models to mine the extremely large data samples. The collaboration between Physicists and Computer scientists will be of paramount importance to develop new algorithms and dedicated solutions to make the output of DL-based DSS interpretable and explainable, which is a deeply felt need in the medical domain [18].

\section{The role of Medical Physicists}

In the AI framework, Medical Physicists have the knowledge needed to implement tests and use imaging systems based on AI $[1,24,66,68]$. They are experts in all imaging modalities and know their strengths and weaknesses. The systematic analysis carried out by Medical Physicists on AI-based DSS systems allows to analyse their response when imaging parameters are modified and, therefore, the quantitative image content is changed [35].

The quantitative information encoded in medical imaging takes on an ever-increasing value as it is correlated to the multiple sources of information contained in the healthcare Big Data (clinical exams, biomarkers, multi-omic data, ecc.). Part of the latter would be available through the access to the EHR. Medical Physicists, taking advantage of the skills acquired by High-Energy Physicists and their experience as data scientists, will be able to derive insights from huge amounts of structured and unstructured data, even using specially designed software, in order to satisfy specific clinical needs (precision medicine).

In this area, Medical Physicists can help improve health services, recognize trends that might otherwise go unnoticed (from the identification of personalized treatments tailored to each individual, to epidemiological studies to promptly analyse the risks to public health), ensure security and compliance. In order for the analyses of this mass of data to be meaningful, they must be properly validated, processed and integrated within a system, something in which the Medical Physicist can make a significant contribution. In fact, Medical Physicists have also a role in the QA analysis of AI-based systems. When an AI tool predicts a machine failure, Medical Physicists can help identify the cause of the issue and corrective actions $[67,69]$.

The added value of Medical Physicists is their capability to go beyond the concept of technical quality and to extend method and expertise toward measuring and optimizing diagnostic value in terms of how it relates to the outcome of care. Moreover, despite the recent advances in the development of Machine Learning, Deep Learning, and the ability of computers to handle large amounts of data, the integration of these technologies into clinical workflows and their application to personalized medicine requires additional research and development efforts.

Large-scale validation of these applications and assessment of the reliability of AI-based systems is needed to facilitate their deployment. A normative framework to regulate the use of AI-based clinical decision 
support tools in the clinical workflow is not available yet. Nevertheless, from the technical point of view we expect that the AI-based tools can be treated similarly to other medical imaging instrumentation and devices. The role of Medical Physicists would be necessary to ensure the quality control of the input data, the periodic evaluation on the DSS performance against possible deterioration due to change in the acquisition protocols for input data, and the quality check on data that can be used in ML and radiomic studies [19].

As reported in Fiorino et al. [68] Medical Physicists are not new to implementing and customizing new systems in a Hospital, usually collaborating with other specialists. In this framework, Medical Physicists must collaborate with all healthcare specialists to successfully introduce AI-based solutions in the clinical workflows. To accomplish this task, Medical Physicists should have a more than basic formation in machine learning, radiomics and the possible source of errors that may hinder the reproducibility of radiomic studies [69,70,71,72,73].

Additionally, Medical Physicists could act as facilitators in the current research challenge of making AI-models explainable, which is a fundamental requirement to make them acceptable as clinical support tools [36]. They are naturally the intermediary professionals between clinicians and technology, so they can help set up the appropriate dictionaries to translate machine information into human (clinical) language and vice versa, thus globally supporting XAI research. Although it is necessary in this case to have a good knowledge of technology it is not necessary to have specific knowledge of ML, DL or other AI techniques. The collaboration with experts working in other fields of research in Physics and in Computer Science is of paramount importance to access to the latest technology and scientific innovations.

Moreover, it is important to highlight the central role of AIFM in this joint project to develop a dedicated infrastructure for medical data analysis with AI. AIFM, through its working groups, could engage the Medical Physics community to facilitate the inclusion of research and clinical groups in joint research activities with INFN using the proposed infrastructure.

\section{Conclusions}

In this paper we focused on the opportunities and challenges provided by Big Data and DL to the medical imaging community, and on the mitigation strategies to the current limitations of these techniques that can be deployed by a joint effort provided by the community of Medical Physicists, High Energy Physicists and Computer Scientists fostered by the agreement between AIFM and INFN.

We proposed the development, deployment and operation of a cloudbased infrastructure to handle and mine big data in the medical imaging domain, especially involving the use of radiomics, ML and DL solutions to set up clinical decision support tools. Solutions and typical use cases are provided for ML model training in substantially different applications, where either original data or derived features may or may not be shared outside the acquisition centres; in this way, data protection and strict privacy policies are built in the model ground-up, using state of the art authentication and authorization tools. Moreover, the data FAIRness compliance of the whole infrastructure would guarantee both the easy validation and cross-check of new results against previously conducted analyses, and the possibility to extend previous studies, thus, fully exploiting the precious resources constituted by patients' data and medical annotations.

Thanks to the healthcare Big Data information, and to its exploitation with the computing infrastructure we proposed it would be possible to extract inferences and useful correlations for predicting the clinical outcome of patients, to identify models that can support the clinicians in the diagnosis or treatment of different diseases, as well as to uncover new pharmacological approaches, the prevention or care of epidemics, maximizing health resources.

Finally, we pointed out that the synergistic effort of a multidisciplinary team of experts in the field of Medical Physics and Data Science would be able to put in place robust AI-based workflows, ready to be validated and used in a clinical setting.

\section{Declaration of Competing Interest}

The authors declare that they have no known competing financial interests or personal relationships that could have appeared to influence the work reported in this paper.

\section{Acknowledgements}

This work has been carried out within the AIM project (Artificial Intelligence in Medicine, AIM, INFN-CSN5, 2019-2021, https://www.pi. infn.it/aim), the ML-INFN project (end-to-end approach to the use of Machine Learning for INFN research lines, ML-INFN, INFN-CSN5, 20202022), the INFN-Cloud initiative, and within the working group on Artificial Intelligence for Medical Physics (AI4MP) of AIFM.

\section{References}

[1] Giger ML, Chan H-P, Boone J. Anniversary Paper: History and status of CAD and quantitative image analysis: The role of Medical Physics and AAPM. Med Phys 2008;35(12):5799-820. https://doi.org/10.1118/1.3013555.

[2] Chan H-P, Doi K, Galhotra S, Vyborny CJ, MacMahon H, Jokich PM. Image feature analysis and computer-aided diagnosis in digital radiography. I. Automated detection of microcalcifications in mammography. Med Phys 1987;14(4):538-48. https://doi.org/10.1118/1.596065.

[3] Kononenko I. Machine learning for medical diagnosis: history, state of the art and perspective. Artif Intell Med 2001;23(1):89-109. https://doi.org/10.1016/S09333657(01)00077-X.

[4] Fujita H. AI-based computer-aided diagnosis (AI-CAD): the latest review to read first. Radiol Phys Technol 2020;13(1):6-19. https://doi.org/10.1007/s12194-01900552-4.

[5] Cui S, Tseng H, Pakela J, Ten Haken RK, El Naqa I. Introduction to machine and deep learning for medical physicists. Med Phys 2020;47:e127-47. https://doi.org/ 10.1002/mp.14140.

[6] Litjens G, Kooi T, Bejnordi BE, Setio AAA, Ciompi F, Ghafoorian M, et al. A survey on deep learning in medical image analysis. Med Image Anal 2017;42:60-88. https://doi.org/10.1016/j.media.2017.07.005.

[7] Chan HP, Hadjiiski LM, Samala RK. Computer-aided diagnosis in the era of deep learning. Med Phys 2020;47:e218-27. https://doi.org/10.1002/mp.13764.

[8] Avanzo M, Porzio M, Lorenzon L, Milan L, Sghedoni R, Russo G, et al. Artificial intelligence applications in medical imaging: A review of the medical physics research in Italy. Phys Medica 2021;83:221-41. https://doi.org/10.1016/j. ejmp.2021.04.010.

[9] Barragán-Montero A, Javaid U, Valdés G, Nguyen D, Desbordes P, Macq B, et al Artificial intelligence and machine learning for medical imaging: A technology review. Phys Medica 2021;83:242-56. https://doi.org/10.1016/j. ejmp.2021.04.016.

[10] Cusumano D, Boldrini L, Dhont J, Fiorino C, Green O, Güngör G, et al. Artificial Intelligence in magnetic Resonance guided Radiotherapy: Medical and physical considerations on state of art and future perspectives. Phys Medica 2021;85: 175-91. https://doi.org/10.1016/j.ejmp.2021.05.010.

[11] Diaz O, Kushibar K, Osuala R, Linardos A, Garrucho L, Igual L, et al. Data preparation for artificial intelligence in medical imaging: A comprehensive guide to open-access platforms and tools. Phys Medica 2021;83:25-37. https://doi.org/ 10.1016/j.ejmp.2021.02.007.

[12] Kalendralis P, Sloep M, van Soest J, Dekker A, Fijten R. Making radiotherapy more efficient with FAIR data. Phys Medica 2021;82:158-62. https://doi.org/10.1016/j. ejmp.2021.01.083.

[13] Ronneberger O, Fischer P, Brox T. U-Net: Convolutional Networks for Biomedical Image Segmentation. Lect. Notes Comput. Sci. (including Subser. Lect. Notes Artif. Intell. Lect. Notes Bioinformatics), vol. 9351, 2015, p. 234-41. https://doi.org/ 10.1007/978-3-319-24574-4_28.

[14] Seo H, Badiei Khuzani M, Vasudevan V, Huang C, Ren H, Xiao R, et al. Machine learning techniques for biomedical image segmentation: An overview of technical aspects and introduction to state-of-art applications. Med Phys 2020;47(5). https://doi.org/10.1002/mp.v47.510.1002/mp.13649.

[15] Gillies RJ, Kinahan PE, Hricak H. Radiomics: Images Are More than Pictures. They Are Data. Radiology 2016;278(2):563-77. https://doi.org/10.1148/ radiol.2015151169.

[16] Avanzo M, Wei L, Stancanello J, Vallières M, Rao A, Morin O, et al. Machine and deep learning methods for radiomics. Med Phys 2020;47(5). https://doi.org/ 10.1002/mp.v47.510.1002/mp.13678.

[17] LeCun Y, Bengio Y, Hinton G. Deep learning. Nature 2015;521(7553):436-44. https://doi.org/10.1038/nature14539.

[18] Vellido A. The importance of interpretability and visualization in machine learning for applications in medicine and health care. Neural Comput Appl 2020;32(24): 18069-83. https://doi.org/10.1007/s00521-019-04051-w. 
[19] Kortesniemi M, Tsapaki V, Trianni A, Russo P, Maas Ad, Källman H-E, et al. The European Federation of Organisations for Medical Physics (EFOMP) White Paper: Big data and deep learning in medical imaging and in relation to medical physics profession. Phys Medica 2018;56:90-3. https://doi.org/10.1016/j. ejmp.2018.11.005.

[20] Manco L, Maffei N, Strolin S, Vichi S, Bottazzi L, Strigari L. Basic of machine learning and deep learning in imaging for medical physicists. Phys Med. 2021;83: 194-205. https://doi.org/10.1016/j.ejmp.2021.03.026.

[21] Castiglioni I, Rundo L, Codari M, Di Leo G, Salvatore C, Interlenghi M, et al. AI applications to medical images: from machine learning to deep learning. Phys Med. 2021;83:9-24. https://doi.org/10.1016/j.ejmp.2021.02.006.

[22] Avanzo M, Trianni A, Botta F, Talamonti C, Stasi M, Iori M. Artificial intelligence and the medical physicist: Welcome to the machine. Appl Sci 2021;11:1-17. https://doi.org/10.3390/app11041691.

[23] Sahiner B, Pezeshk A, Hadjiiski LM, Wang X, Drukker K, Cha KH, et al. Deep learning in medical imaging and radiation therapy. Med Phys 2019;46(1):e1-36. https://doi.org/10.1002/mp.2019.46.issue-110.1002/mp.13264.

[24] Jarrett D, Stride E, Vallis K, Gooding MJ. Applications and limitations of machine learning in radiation oncology. Br J Radiol 2019;92(1100):20190001. https://doi. org/10.1259/bjr.20190001.

[25] Thompson MK, Poortmans P, Chalmers AJ, Faivre-Finn C, Hall E, Huddart RA, et al. Practice-changing radiation therapy trials for the treatment of cancer: where are we 150 years after the birth of Marie Curie? Br J Cancer 2018;119(4):389-407. https://doi.org/10.1038/s41416-018-0201-z.

[26] Siddique S, Chow JCL. Artificial intelligence in radiotherapy. Reports Pract Oncol Radiother 2020;25(4):656-66. https://doi.org/10.1016/j.rpor.2020.03.015.

[27] McNutt TR, Benedict SH, Low DA, Moore K, Shpitser I, Jiang W, et al. Using Big Data Analytics to Advance Precision Radiation Oncology. Int J Radiat Oncol 2018; 101(2):285-91. https://doi.org/10.1016/j.ijrobp.2018.02.028.

[28] Kalet AM, Luk SMH, Phillips MH. Radiation Therapy Quality Assurance Tasks and Tools: The Many Roles of Machine Learning. Med Phys 2020;47:e168-77. https:// doi.org/10.1002/mp.13445.

[29] Chan MF, Witztum A, Valdes G. Integration of AI and Machine Learning in Radiotherapy QA. Front Artif Intell 2020;3:1-8. https://doi.org/10.3389/ frai.2020.577620.

[30] Greenspan H, van Ginneken B, Summers RM. Guest Editorial Deep Learning in Medical Imaging: Overview and Future Promise of an Exciting New Technique. IEEE Trans Med Imaging 2016;35(5):1153-9. https://doi.org/10.1109/ TMI.2016.2553401.

[31] He J, Baxter SL, Xu J, Xu J, Zhou X, Zhang K. The practical implementation of artificial intelligence technologies in medicine. Nat Med 2019;25(1):30-6. https:// doi.org/10.1038/s41591-018-0307-0.

[32] Wilkinson MD, Dumontier M, Aalbersberg IJ, Appleton G, Axton M, Baak A, et al. The FAIR Guiding Principles for scientific data management and stewardship. Sci Data 2016;3(1). https://doi.org/10.1038/sdata.2016.18.

[33] Retico A, Arezzini S, Bosco P, Calderoni S, Ciampa A, Coscetti S, et al. ARIANNA: A research environment for neuroimaging studies in autism spectrum disorders. Comput Biol Med 2017;87:1-7. https://doi.org/10.1016/j. compbiomed.2017.05.017.

[34] Balagurunathan Y, Mitchell R, El Naqa I. Requirements and reliability of AI in the medical context. Phys Medica 2021;83:72-8. https://doi.org/10.1016/j. ejmp.2021.02.024.

[35] Mahadevaiah G, RV P, Bermejo I, Jaffray D, Dekker A, Wee L. Artificial intelligence-based clinical decision support in modern medical physics: Selection, acceptance, commissioning, and quality assurance. Med Phys 2020;47(5). https:/ doi.org/10.1002/mp.v47.510.1002/mp.13562.

[36] McCarthy N, Dahlan A, Cook TS, Hare NO', Ryan M-L, St John B, et al. Enterprise imaging and big data: A review from a medical physics perspective. Phys Medica 2021;83:206-20. https://doi.org/10.1016/j.ejmp.2021.04.004.

[37] Markus AF, Kors JA, Rijnbeek PR. The role of explainability in creating trustworthy artificial intelligence for health care: A comprehensive survey of the terminology, design choices, and evaluation strategies. J Biomed Inform 2021;113:103655. https://doi.org/10.1016/j.jbi.2020.103655.

[38] Amann J, Blasimme A, Vayena E, Frey D, Madai VI. Explainability for artificial intelligence in healthcare: a multidisciplinary perspective. BMC Med Inform Decis Mak 2020;20:310. https://doi.org/10.1186/s12911-020-01332-6.

[39] Lombardi A, Diacono D, Amoroso N, Monaco A, Tavares JMRS, Bellotti R, et al. Explainable Deep Learning for Personalized Age Prediction With Brain Morphology. Front Neurosci 2021;15. https://doi.org/10.3389/ fnins.2021.67405510.3389/fnins.2021.674055.s001.

[40] Beckers R, Kwade Z, Zanca F. The EU medical device regulation: Implications for artificial intelligence-based medical device software in medical physics. Phys Medica 2021;83:1-8. https://doi.org/10.1016/j.ejmp.2021.02.011.

[41] Gagliardi F, Jones B, Reale M, Burke S. European DataGrid Project: Experiences of Deploying a Large Scale Testbed for E-science Applications. Lect. Notes Comput. Sci. (including Subser. Lect. Notes Artif. Intell. Lect. Notes Bioinformatics), vol. 2459, 2002, p. 480-99. https://doi.org/10.1007/3-540-45798-4_20.

[42] Gagliardi F. The EGEE European grid infrastructure project. Lect Notes Comput Sci 2005;3402:194-203. https://doi.org/10.1007/11403937 16.

[43] Salomoni D, Campos I, Gaido L, de Lucas JM, Solagna P, Gomes J, et al. INDIGODataCloud: a Platform to Facilitate Seamless Access to E-Infrastructures. J Grid Comput 2018;16(3):381-408. https://doi.org/10.1007/s10723-018-9453-3.

[44] Espinal X, Jezequel S, Schulz M, Sciabà A, Vukotic I, Wuerthwein F, et al. The Quest to solve the HL-LHC data access puzzle. EPJ Web Conf 2020;245:04027. https://doi.org/10.1051/epjconf/202024504027.
[45] Ceccanti A, Hardt M, Wegh B, Millar AP, Caberletti M, Vianello E, et al. The INDIGO-Datacloud Authentication and Authorization Infrastructure. J Phys Conf Ser 2017;898:102016. https://doi.org/10.1088/1742-6596/898/10/102016.

[46] Garonne V, Vigne R, Stewart G, Barisits M, eermann TB, Lassnig M, et al. Rucio-the next generation of large scale distributed system for ATLAS Data Management. J Phys Conf Ser 2014;513(4):042021. https://doi.org/10.1088/1742-6596/513/ 4/042021.

[47] Jochems A, Deist TM, van Soest J, Eble M, Bulens P, Coucke P, et al. Distributed learning: Developing a predictive model based on data from multiple hospitals without data leaving the hospital - A real life proof of concept. Radiother Oncol 2016;121(3):459-67. https://doi.org/10.1016/j.radonc.2016.10.002.

[48] Deist TM, Jochems A, van Soest J, Nalbantov G, Oberije C, Walsh S, et al. Infrastructure and distributed learning methodology for privacy-preserving multicentric rapid learning health care: euroCAT. Clin Transl Radiat Oncol 2017;4: 24-31. https://doi.org/10.1016/j.ctro.2016.12.004.

[49] Ashburner J. A fast diffeomorphic image registration algorithm. Neuroimage 2007; 38(1):95-113. https://doi.org/10.1016/j.neuroimage.2007.07.007.

[50] Ferrari E, Bosco P, Calderoni S, Oliva P, Palumbo L, Spera G, et al. Dealing with confounders and outliers in classification medical studies: The Autism Spectrum Disorders case study. Artif Intell Med 2020;108:101926. https://doi.org/10.1016/ j.artmed.2020.101926.

[51] M. Hoogman D. Rooij M. Klein P. Boedhoe I. Ilioska T. Li et al. Consortium neuroscience of attention deficit/hyperactivity disorder and autism spectrum disorder: The $<$ scp $>$ ENIGMA $</$ scp $>$ adventure Hum Brain Mapp 2020:hbm.25029. $10.1002 / \mathrm{hbm} .25029$.

[52] Lizzi F, Agosti A, Brero F, Cabini RF, Fantacci ME, Figini S, et al. Quantification of pulmonary involvement in COVID-19 pneumonia by means of a cascade oftwo Unets: training and assessment on multipledatasets using different annotation criteria. https://doi.org/10.1007/s11548-021-02501-2.

[53] Ronneberger O, Fischer P, Brox T. U-net: Convolutional networks for biomedical image segmentation. Lect Notes Comput Sci (Including Subser Lect Notes Artif Intell Lect Notes Bioinformatics) 2015;9351:234-41. https://doi.org/10.1007/ 978-3-319-24574-4_28.

[54] Rigby MJ. Ethical Dimensions of Using Artificial Intelligence in Health Care. AMA J Ethics 2019;21:E121-4. https://doi.org/10.1001/amajethics.2019.121.

[55] Gray M, Lagerberg T, Dombrádi V. Equity and value in 'Precision Medicine'. New Bioeth 2017;23(1):87-94. https://doi.org/10.1080/20502877.2017.1314891.

[56] Shaban-Nejad A, Michalowski M, Peek N, Brownstein JS, Buckeridge DL. Seven pillars of precision digital health and medicine. Artif Intell Med 2020;103:101793. https://doi.org/10.1016/j.artmed.2020.101793.

[57] Cerello P, Bagnasco S, Bottigli U, Cheran SC, Delogu P, Fantacci ME, et al. GPCALMA: a Grid-based tool for mammographic screening. Methods Inf Med 2005; 44(02):244-8.

[58] Bellotti R, De Carlo F, Tangaro S, Gargano G, Maggipinto G, Castellano M, et al. A completely automated CAD system for mass detection in a large mammographic database. Med Phys 2006;33(8):3066-75. https://doi.org/10.1118/1.2214177.

[59] van Ginneken B, Armato SG, de Hoop B, van Amelsvoort-van de Vorst S, Duindam T, Niemeijer M, et al. Comparing and combining algorithms for computer-aided detection of pulmonary nodules in computed tomography scans: The ANODE09 study. Med Image Anal 2010;14(6):707-22. https://doi.org/ 10.1016/j.media.2010.05.005.

[60] Retico A, Bosco P, Cerello P, Fiorina E, Chincarini A, Fantacci ME. Predictive Models Based on Support Vector Machines: Whole-Brain versus Regional Analysis of Structural MRI in the Alzheimer's Disease. J Neuroimaging 2015;25(4):552-63. https://doi.org/10.1111/jon.12163.

[61] Donatelli G, Retico A, Caldarazzo Ienco E, Cecchi P, Costagli M, Frosini D, et al. Semiautomated Evaluation of the Primary Motor Cortex in Patients with Amyotrophic Lateral Sclerosis at 3T. Am J Neuroradiol 2018;39(1):63-9. https://doi. org/10.3174/ajnr.A5423.

[62] Montalt-Tordera J, Muthurangu V, Hauptmann A, Steeden JA. Machine learning in magnetic resonance imaging: image reconstruction. Phys Med. 2021;83:79-87. https://doi.org/10.1016/j.ejmp.2021.02.020.

[63] Papadimitroulas P, Brocki L, Christopher Chung N, Marchadour W, Vermet F, Gaubert L, et al. Artificial intelligence: deep learning in oncological radiomics and challenges of interpretability and data harmonization. Phys Med. 2021;83:108-21.

[64] Chatrchyan S, Hmayakyan G, Khachatryan V, Sirunyan AM, Adam W, Bauer T, et al. The CMS experiment at the CERN LHC. J Instrum 2008;3:S08004-S08004. https://doi.org/10.1088/1748-0221/3/08/S08004.

[65] Radovic A, Williams M, Rousseau D, Kagan M, Bonacorsi D, Himmel A, et al. Machine learning at the energy and intensity frontiers of particle physics. Nature 2018;560(7716):41-8. https://doi.org/10.1038/s41586-018-0361-2.

[66] El Naqa I, Das S. The role of machine and deep learning in modern medical physics. Med Phys 2020;47:e125-6. https://doi.org/10.1002/mp.14088.

[67] Wang B, White G. The role of clinical medical physicists in the future: Quality, safety, technology implementation, and enhanced direct patient care. J Appl Clin Med Phys 2019;20(6):4-6. https://doi.org/10.1002/acm2.2019.20.issue610.1002/acm2.12619.

[68] Fiorino C, Jeraj R, Clark CH, Garibaldi C, Georg D, Muren L, et al. Grand challenges for medical physics in radiation oncology. Radiother Oncol 2020;153:7-14. https://doi.org/10.1016/j.radonc.2020.10.001.

[69] Yip SSF, Aerts HJWL. Applications and limitations of radiomics. Phys Med Biol 2016;61(13):R150-66. https://doi.org/10.1088/0031-9155/61/13/R150.

[70] Traverso A, Wee L, Dekker A, Gillies R. Repeatability and reproducibility of radiomic features: A systematic review. Int J Radiat Oncol 2018;102(4):1143-58. https://doi.org/10.1016/j.ijrobp.2018.05.053. 
[71] Diaz O, Guidi G, Ivashchenko O, Colgan N, Zanca F. Artificial intelligence in the medical physics community: An international survey. Phys Med 2021;(81):141-6. https://doi.org/10.1016/j.ejmp.2020.11.037.

[72] Doria S, Valeri F, Lasagni L, Sanguineti V, Ragonesi R, Akbar MU, et al. Addressing signal alterations induced in CT images by deep learning processing: a preliminary phantom study. Phys Med. 2021;83:88-100. https://doi.org/10.1016/j. ejmp.2021.02.022.

[73] Nousiainen K, Mäkelä T, Piilonen A, Peltonen JI. Automating chest radiograph imaging quality control. Phys Med. 2021;83:138-45. https://doi.org/10.1016/j. ejmp.2021.03.014.

Alessandra Retico ${ }^{\mathrm{a}}$, Michele Avanzo ${ }^{\mathrm{b}}$, Tommaso Boccali ${ }^{\mathrm{a}}$, Daniele Bonacorsi $^{\mathrm{c}, \mathrm{d}}$, Francesca Botta ${ }^{\mathrm{e}}$, Giacomo Cuttone ${ }^{\mathrm{f}}$, Barbara Martelli $^{g}$, Davide Salomoni ${ }^{g}$, Daniele Spiga ${ }^{\mathrm{h}}$, Annalisa Trianni, Michele Stasi ${ }^{j}$, Mauro Iori ${ }^{\mathrm{k}, *}$, Cinzia Talamonti ${ }^{1, \mathrm{~m}}$

${ }^{a}$ National Institute for Nuclear Physics (INFN), Pisa Division, 56127 Pisa, Italy

${ }^{\mathrm{b}}$ Medical Physics Department, Centro di Riferimento Oncologico di Aviano (CRO) IRCCS, 33081 Aviano, Italy ${ }^{\mathrm{c}}$ University of Bologna, 40126 Bologna, Italy d INFN, Bologna Division, 40126 Bologna, Italy
${ }^{\mathrm{e}}$ Medical Physics Unit, Istituto Europeo di oncologia IRCCS, 20141 Milan,

Italy

${ }^{\mathrm{f}}$ INFN, Southern National Laboratory (LNS), 95123 Catania, Italy

${ }^{\mathrm{g}}$ INFN, CNAF Division, 40126 Bologna, Italy

${ }^{\mathrm{h}}$ INFN, Perugia Division, 06123 Perugia, Italy

${ }^{i}$ Medical Physics Unit, Ospedale Santa Chiara APSS, 38122 Trento, Italy

${ }^{j}$ Medical Physics Unit, A.O. Ordine Mauriziano di Torino, 10128 Torino,

Italy

Emilia, Italy

${ }^{1}$ Department Biomedical Experimental and Clinical Science "Mario Serio", University of Florence, 50134 Florence, Italy ${ }^{\mathrm{m}}$ INFN, Florence Division, 50134 Florence, Italy

* Corresponding author at: Medical Physics Unit, Azienda USL-IRCCS di Reggio Emilia, 42122 Reggio Emilia, Italy. E-mail address: mauro.iori@ausl.re.it (M. Iori). 